\title{
Becoming Certain About the Uncertain: How AI Changes Proof-of-Concept Activities in Manufacturing - Insights from a Global Automotive Leader
}

\author{
André Sagodi \\ University of St.Gallen \\ andre.sagodi@student.unisg.ch \\ Johannes Schniertshauer \\ AUDI AG \\ johannes.schniertshauer@audi.de
}

\author{
Christian Engel \\ University of St.Gallen \\ christian.engel@unisg.ch \\ Benjamin van Giffen \\ University of St.Gallen \\ benjamin.vangiffen@unisg.ch
}

\begin{abstract}
In this paper, we examine Proof-of-Concept activities in the presence of Artificial Intelligence (AI). To this end, we conducted an exploratory, revelatory case study at a leading automotive OEM that constantly explores new technologies to improve its manufacturing processes. We highlight how AI properties affect specifics in project execution and how they are addressed within the focal company. We carved out four key areas affecting underlying activities, i.e., data assessment, process alignment, value orientation, and AI empowerment. With our findings, we provide practical insights into AI-related challenges and corresponding pathways for action. Drawn upon, we develop novel, timely, and actionable recommendations for AI project leaders planning to implement this novel technology in manufacturing. This shall provide empirically grounded and conceptually sound guidance for researchers and practitioners alike, and ultimately foster the success of AI in manufacturing.
\end{abstract}

\section{Introduction}

Since the first industrial revolution, waves of technological innovations have transformed manufacturing companies [1]. These changes were mainly driven by organizations that incorporate technologies to increase efficiency, improve decision making, or create new business opportunities [2]. Growing computing power and Big Data gave rise to AI technologies, hence it is today a fixed point on the agenda of many companies aiming to leverage $\mathrm{AI}$ in their respective business context [3,4]. AI allows for automating functions that are vastly associated with the human mind, such as sensing, perceiving, interacting with the environment, problem-solving, learning, or decision-making $[5,6]$. $\mathrm{AI}$ is a "misleadingly used [...] umbrella term" (p. 3), nowadays mainly referring to applications based on Machine Learning [7], and provides vast innovation opportunities for companies, promising to enable the automation of cognitive tasks. As AI advances are fueling the pace of automation [8], this worldwide trend is expected to cause great business impact for companies across all industries [9]. Our paper focuses on the manufacturing industry, where the degree of automation is estimated to increase from globally $10 \%$ to around 25\% [5]. Combined with the increasing availability of data, this is likely to impact the workforce more fundamentally than the first industrial revolution [10].

However, while technological progress seems limitless, new applications can face new barriers and unprecedented challenges [11]. For instance, it is reported that many of the current AI initiatives fail [12], and that the initial hype and accompanying high expectations have now been replaced by a more pessimistic and sober view towards AI [13]. If manufacturing organizations intend to benefit from AI, they need a sophisticated understanding of the tools and associated challenges, e.g., to avoid the pitfall of underestimating its potential costs [14]. Hence, it is decisive to gain further insights on how to establish new value creation mechanisms with AI, and, therefore, to evaluate AI particularly in the manufacturing context, i.e., in terms of specific tasks, and compared to alternative investments [14]. In this gap between value expectation and value realization, Proof-of-Concepts (PoCs) play an important role for manufacturing organizations when it comes to engaging with new technologies and understanding them in their own business context. Previous literature revealed that the specific characteristics of $\mathrm{AI}$ challenge previous technology selection processes and 
for successful technology implementation, companies need to explicitly consider the specifics of AI [15]. Against this backdrop, the role of PoCs changes due to the distinguishable technological properties of AI, e.g., its experimental character, black box characteristics, context sensitivity, and learning requirements [16]. Accordingly, the goal of this paper is to support AI project leaders in their AI PoC activities based on solid, empirical, real-world insights. For this occasion, we pose the following research question: How do AI properties affect the PoC activities in manufacturing organizations? To answer this research question, we conducted an exploratory, revelatory case study at a leading automotive OEM that explores AI technologies to improve its production processes and make them more efficient.

\section{Conceptual Background}

\subsection{Implementing AI in Organizations}

AI is a general-purpose technology that provides a wide variety of potential opportunities for organizations. It can be characterized along the following properties, which research has identified [16]: (1) experimental character, (2) context sensitivity, (3) black box character, and (4) learning requirements. We briefly summarize them as follows: (1) The experimental character of AI refers to the outcomes of AI systems being probabilistic rather than deterministic, i.e., do not follow rule-based "if-then" structures [17]; (2) the context sensitivity refers to AI systems' performance depending on the data their context provides to explain and predict this context [18]; (3) the black box character refers to AI systems, especially in the field of deep learning, facing challenges in delivering explanations to humans on what happens between data input and AI output [19]; (4) the learning requirements of AI refer to the need for AI solutions to learn and develop experience from data-based examples with the goal to improve their performance over time [20].

These AI properties are facilitated by the advancements in computing power and the pervasiveness of data (storage) [3, 4]. On the one hand they facilitate novel opportunities for organizations, on the other hand, they induce novel technology implementation challenges into organizations. This means that mere technological advancements are not causal to business goal achievement [21] and "while technology advancements may have no limit, its applications may encounter bottlenecks and unprecedented barriers" ([11], p. 69). Thus, effectively ensuring the fit between technologies and business processes in given organizational contexts makes the difference between an organization's success or failure [22]. With AI being context-sensitive, this is even more relevant for highly specialized environments, since in many cases, such as in highly specialized manufacturing, it is not possible to simply buy readymade plug-and-play AI solutions. Hence, understanding the technology and implementing it in a value-adding way is a vital capability for organizations.

Research on AI implementation is nascent but quickly emerging. This is reflected in recent calls for research on how different AI technologies can be integrated within organizations (e.g., [5]). In the same vein, Loureiro et al. [23] raise the question: "[h]ow should organizations manage and implement AI systems in their organizations?" (p. 921), and Duan et al. [11] highlight the need to identify critical success factors for the implementation of effective and acceptable AI applications based on empirical evidence, which then can provide guidance for organizations to focus on most critical aspects. On that note, PoCs as an activity in the early phases of AI implementation projects play a particularly crucial role and have the potential to support organizational decision-makers define their AI project portfolios. This then also benefits investment decisions, resource allocation, and portfolio risk management.

\subsection{Proof-of-Concept in IS Research}

Neto et al. [24] revealed that PoC practices are largely under-researched in scientific literature. PoCs are means to demonstrate that a potential solution is functionally feasible [25]. In the Oxford English Dictionary, a PoC is broadly defined as "evidence (usually deriving from an experiment or pilot project) demonstrating that a design concept, business idea, etc., is feasible" ([26], p. 737). On a more abstract level, a PoC can be understood as a research practice aiming to generate new knowledge through experimental tests [27]. From a different angle, PoC activities aim to broaden problem understanding and provide space for scientific activities to create new knowledge that informs further design decisions, hence contribute to the future feasibility of a solution [25]. In this paper, we adopt the definition proposed by Neto et al. [24] and define a PoC as "an activity system [...] in a socio-technical context, with the purpose of evaluation, understanding, validation and exploration, and with the aim of learning about technological artifacts and their phenomena under study by organizations and PoC practitioners" (p. 2). 


\subsection{The Need for Empirical Research on AI PoCs in Manufacturing}

PoCs are important for organizations for making sense of new (technological) opportunities. Organizations conduct PoCs to gain new knowledge and to validate new concepts, e.g., through test setups, simulations, or analyses. This poses a strategic organizational capability, which can make the difference between successful and failing companies in volatile, uncertain, complex, and ambiguous environments [28]. When it comes to making sense of $\mathrm{AI}$, it is important to consider that the unique AI properties require novel considerations prior to its implementation in business processes. It can therefore be assumed that, within AI PoCs, other or adapted activities need to be addressed, which then lead to relevant knowledge on AI-specific aspects.

Based on the introduction of AI properties in the prior section, we present a high-level reasoning of how these properties affect PoCs, which is further empirically substantiated in this study: First, AI is context-sensitive, which imposes the need to rethink PoCs, moving from a merely technical feasibility perspective to one that accounts for the organizational embedding. Second, AI has learning requirements as AI algorithms are trained rather than programmed. This leads to a shift in risk distribution over time as once all the data is gathered, cleaned, and fed into the algorithmic models, a large share of the project effort is already done. This requires novel approaches for designing, scoping, and timing PoCs for AI. Third, this largely relates to the experimental character of AI, which means that non-deterministic results are produced in a manner of probabilism. This changes the notion of PoCs from "this system works" to "this system likely works", which imposes novel challenges on project (portfolio) planning, risk, and stakeholder management. Hence, the advent of AI with its novel properties imposes novel PoC implementation barriers on organizations. ID01: "[T] his high uncertainty at the beginning as well as low success rates are such a main differentiator [of $\mathrm{AI} \mathrm{PoCs}$ ]"

However, so far there has been no sound and empirically grounded research on PoCs for AI implementation in manufacturing organizations. Therefore, we examine the cause-effect relationships of $\mathrm{AI}$ characteristics and the $\mathrm{AI}$ PoC activities in the context of manufacturing.

\section{Research Design: In-Depth Case Study}

Our research aims to identify AI-induced $\mathrm{PoC}$ activities - a new phenomenon in a contemporary reallife context. Therefore, a single case study sets a suitable foundation [29, 30]. We engaged in an exploratory qualitative case study by conducting semistructured interviews within a leading global original equipment manufacturer (OEM). Such an approach is suitable given the novelty of the topic, lack of theory, and its relevance for practice. By selecting an industry leader, we aim to base our research insights on a revelatory case foundation. In doing so, we also account for the context-dependence of PoC activities in AI implementation projects.

\subsection{Case Selection}

The manufacturing context of an automotive company is suitable for this research due to the topicality of the subject in the industry. For example, Winkler et al. [13] conclude that automotive companies are still facing AI implementation challenges and strive to deduce business value from PoCs. Automotive companies are under constant cost pressure and consistently strive to improve their manufacturing efficiency. As a result, tinkering and making sense of emerging technologies is a constant imperative for staying competitive in the automotive industry. In the German-speaking area, the latest technological movement is referred to as 'Industry 4.0' and describes "primarily IT driven [...] changes in manufacturing systems" ([31], p. 241).

We seized the unique opportunity to collect our data in the context of a leading global automotive OEM (Original Equipment Manufacturer) that we refer to as 'Automotive AG'. The case company has already successfully deployed AI as well as employs AI experts and data scientists. Moreover, Automotive AG stands on the edge of implementing AI-based Computer Vision in its manufacturing context on a larger scale. A recent study shows that practitioners assess the use of AI-based Computer Vision for, e.g., visual quality control, as both highly realizable and highly valuable [32], what we acknowledge in our data collection strategy.

\subsection{Data Collection and Analysis}

The research team had comprehensive access to company data which enabled the use of multiple data sources, as well as triangulation of findings during data analysis. Data sources included on-site visits, interviews, and observations (within heterogeneous groups), secondary data (e.g., internal presentations, and public statements), as well as domain expertise within the research team.

All interviewed practitioners were involved in at least one AI PoC thus could provide first-hand information. Altogether, the experience of the 
interviewees spans a multitude of cases within the situated organizational context. As summarized in Table 1, we purposefully selected three cases in detail, as well as a collection of early AI PoC ideas to allow for a high level of variation between AI PoC cases. Furthermore, the cross-case analysis of distinct AI PoCs bases our empirical research insights on a broader conceptual basis.

\section{Table 1. Scope of focal AI PoCs}

\section{\#1 - Crack detection}

Implementation of a prototype to detect cracks on deep-drawn parts in the press line using Convolutional Neural Networks (CNN) to assist workers and reduce manual effort.

\#2 - Welding point quality inspection

Integration of a condition monitoring system based on Supervised Learning algorithms that analyzes various sensor data to detect quality problems and optimize welding parameters of robots.

\#3 - Tire information readout

Building a prototype to localize labels on vehicle tires using CNNs, and processing information using state-of-the-art Optical Character Recognition (OCR) algorithms to document information according to legal requirements. \#4 - Early AI PoC ideas

Exploration of further use case ideas at an early AI PoC stage. This particularly refers to the implementation of AI-based Computer Vision solutions, mainly in the field of quality assurance.

We conducted 12 semi-structured interviews with experts on the four focal AI PoC cases (see Table 2). Conducting the interviews allowed for a natural flow and to adapt the conversation to emerging themes. All interviews were conducted by the authors. To substantiate our data collection, we participated in several PoC meetings and reviewed case documents made available before and after the interviews.

Table 2. Overview of data collection

\begin{tabular}{|c|c|c|c|c|c|c|}
\hline \multirow[t]{2}{*}{ ID } & \multirow[t]{2}{*}{ Position } & \multicolumn{4}{|c|}{ \# Focal AI PoCs } & \multirow[t]{2}{*}{ Duration } \\
\hline & & 1 & 2 & 3 & 4 & \\
\hline 01 & ML Engineer & $\mathrm{x}$ & $\mathrm{x}$ & & & $65 \mathrm{~min}$ \\
\hline 02 & Data Scientist & & & $\mathrm{x}$ & & $60 \mathrm{~min}$ \\
\hline 03 & Data Analyst & $\mathrm{x}$ & $\mathrm{x}$ & & & $70 \mathrm{~min}$ \\
\hline 04 & Product Owner & $\mathrm{x}$ & & & & $135 \mathrm{~min}$ \\
\hline 05 & Product Owner & & $\mathrm{x}$ & & & $30 \mathrm{~min}$ \\
\hline 06 & Data Scientist & & $\mathrm{x}$ & & & $60 \mathrm{~min}$ \\
\hline 07 & Digitalization Expert & & & & $\mathrm{x}$ & $60 \mathrm{~min}$ \\
\hline 08 & Data Analyst & & & $\mathrm{x}$ & & $50 \mathrm{~min}$ \\
\hline 09 & Technology Manager & $\mathrm{x}$ & $\mathrm{x}$ & $\mathrm{x}$ & & $55 \mathrm{~min}$ \\
\hline 10 & Technology Expert & & & & $\mathrm{x}$ & $55 \mathrm{~min}$ \\
\hline 11 & Technology Expert & & & $\mathrm{x}$ & $\mathrm{x}$ & $55 \mathrm{~min}$ \\
\hline 12 & Digitalization Expert & & & & $\mathrm{x}$ & $75 \mathrm{~min}$ \\
\hline \multicolumn{6}{|c|}{ 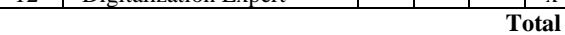 } & $12 \mathrm{~h} 50 \mathrm{~min}$ \\
\hline \multicolumn{7}{|c|}{$\begin{array}{l}\# 1 \text { - Crack detection } \\
\# 2 \text { - Welding point quality inspection } \\
\# 3 \text { - Tire information readout } \\
\# 4 \text { - Early AI PoC ideas }\end{array}$} \\
\hline
\end{tabular}

Our data analysis followed a three-step coding process [33] that comprised (1) open coding, (2) axial coding, and (3) selective coding to elaborate on the effects of AI properties on AI PoC activities at Automotive AG. In the (1) open-coding stage, codes emerged through case write-ups and summaries which were used to condense the transcripts to obtain an initial overview of all case data [30]. In the (2) axial coding stage, the research team condensed all data based on recurring themes. Finally, (3) selective coding allowed to sharpen the focus on AI PoC challenges at Automotive AG. To analyze and manage the collected data systematically, we used ATLAS.ti as our qualitative data analysis software. During coding, the research team triangulated the insights with obtained results from analyzing the internal (e.g., internal presentations) and external (e.g., public statements) case material. In cases of disagreement, the research team could reconnect with the interviewees to validate its findings, or to gather additional case materials.

\section{Case Study Results: Tackling Challenges of AI PoCs at Automotive AG}

\subsection{Case Background and Drivers of AI PoCs at Automotive Corp}

Automotive AG's customers can individualize their vehicles and select amongst a wide range of optional features such as autonomous driving functions. This individualization leads to increasing product (portfolio) variance, which Automotive AG must handle in its manufacturing context. To operate efficiently in this increasingly complex context, flexible automation solutions and the digitization of products and processes are decisive competitive factors. For this reason, Automotive AG nowadays heavily explores data-driven technologies such as AI. To test and prove how to deploy AI technologies in a value-adding manner into manufacturing, PoCs are an essential means at the focal company, as they facilitate the creation of organizational knowledge, build relevant AI skills, and validate potential use cases at an early stage. Hence, AI PoCs enable Automotive AG to conduct more accurate cost estimations, and to build a value-oriented AI portfolio. For Automotive AG, developing and testing novel technologies such as AI in PoCs before deploying them in high-performance manufacturing environments is a critical capability and competitive differentiator.

\subsection{Challenges in the Implementation of AI PoCs at Automotive AG}

In this section, we present four identified clusters of AI PoC challenges (data assessment, process alignment, value orientation, and AI empowerment) and the respective counterstrategies (pathways for action) derived from the analysis of the Automotive AG case (see Table 3). We further map our findings to the related AI properties that induce the AI PoC challenges: experimental character $(\mathrm{E})$, black box 
characteristics (B), context sensitivity (C), and learning requirements (L). In doing so, we provide empirical evidence on why and how these challenges formed. In the following subchapters, we elaborate on the pathways for action with reference to our case.

Table 3. Challenges and Pathways for Action

\begin{tabular}{|c|c|}
\hline $\begin{array}{l}\text { AI PoC challenges } \\
\text { (related AI properties) }\end{array}$ & Pathways for action in AI PoCs \\
\hline \multicolumn{2}{|l|}{ Data assessment } \\
\hline $\begin{array}{l}\text { Infrastructure and } \\
\text { automation }(\mathrm{L})\end{array}$ & \multirow{3}{*}{$\begin{array}{l}\text { - Deploy a digital platform to harmonize data } \\
\text { from all manufacturing sites } \\
\text { - Implement a detailed process model for } \\
\text { collecting data in a reproducible manner } \\
\text { - Use data checklists as self-assessment tools to } \\
\text { identify blind spots early } \\
\text { - Use synthetic data to train algorithms } \\
\text { - Qualify a team of internal factory workers as } \\
\text { full-time data annotators }\end{array}$} \\
\hline Data collectability $(\mathrm{L})$ & \\
\hline Data labeling (C) & \\
\hline \multicolumn{2}{|l|}{ Process alignment } \\
\hline Problem-centricity (B) & \multirow{3}{*}{$\begin{array}{l}\text { - Embed the PoC in an existing production } \\
\text { business scenario (e.g., consider lean principles) } \\
\text { - Analyze the processes before getting started } \\
\text { - Involve AI experts early in the PoC definition } \\
\text { phase in a consulting role }\end{array}$} \\
\hline $\begin{array}{l}\text { Business process } \\
\text { mapping }(\mathrm{C})\end{array}$ & \\
\hline $\begin{array}{l}\text { Mathematical problem } \\
\text { definition (E) }\end{array}$ & \\
\hline \multicolumn{2}{|l|}{ Value orientation } \\
\hline $\begin{array}{l}\text { Use case simplification } \\
\text { (C) }\end{array}$ & \multirow{3}{*}{$\begin{array}{l}\text { - Define the actual technical-mathematical } \\
\text { question or hypothesis to be retrieved } \\
\text { - Deploy agile project management principles } \\
\text { - Adapt a comprehensive guide for potential } \\
\text { customers to understand the metrics used to } \\
\text { evaluate the algorithms in place }\end{array}$} \\
\hline $\begin{array}{l}\text { Business case } \\
\text { unpredictability (E) }\end{array}$ & \\
\hline Success metrics (E) & \\
\hline \multicolumn{2}{|l|}{ AI empowerment } \\
\hline Demystification (B) & \multirow{3}{*}{$\begin{array}{l}\text { - Offer AI training on an organizational level } \\
\text { - Present and discuss use cases in tech talks } \\
\text { - Create a common understanding of AI right in } \\
\text { the definition phase of the AI PoC } \\
\text { - Involve subject matter experts early } \\
\text { - Install innovation hubs } \\
\text { - Explore AI under near-production conditions }\end{array}$} \\
\hline $\begin{array}{l}\text { Customer enablement } \\
\text { (L) }\end{array}$ & \\
\hline Capability building (B) & \\
\hline $\boldsymbol{E}$ - experimental char & $\begin{array}{l}\text { acter; } \boldsymbol{B} \text { - black box characteristics; } \boldsymbol{C} \text { - context } \\
\text { requirements }\end{array}$ \\
\hline
\end{tabular}

4.2.1 Data Assessment. First, learning requirements of AI induce a challenge in the realm of infrastructure and automation. Ten interviewees stated that the lack of automated solutions for ingesting streaming or batch processing data from relevant sources is a challenge, and to make it accessible through a centralized storage, e.g., data lake. In the crack detection case (\#1) it became obvious, during the collection of images showing cracked parts to train an algorithm, that creating an image dataset by hand was not manageable and would have resulted in an excessive workload. ID04: "Even if the images are on a drive and you must take them somewhere else for labeling or processing, which can be very time consuming, you realize more and more that it is absolutely necessary that everything in the data acquisition is automated: from image capturing to storing examples in a database [...] and because of all this data handling, it happens often that you suddenly have duplicates in there." Automotive AG solved this challenge by developing a dedicated manufacturing cloud platform to harmonize data from all global manufacturing sites. This platform is used as technological basis for most of the analyzed use cases and made it possible to combine data from various domains for complex automation ideas. Second, the learning requirements of $\mathrm{AI}$ induce data collectability challenges. This refers to the need for data to be technically collectable from the manufacturing context to conduct an AI PoC. As stated by ID03, manual processes cannot be automated without implementing data acquisition tools such as cameras to capture the required images for training AI algorithms: "So from that point of view, data quality and data availability are clearly an issue because otherwise I can't perform a PoC in the simplest case. If I can't access the data and if it's not available to me then it won't be in a later industrialized state either." In many cases, the first step in a successful AI PoC is therefore not to train a deep-learning model, but to set up and deploy the tools required for collecting the dataset. Even when data can be captured from a technological perspective, the specific characteristics of industrial data can be challenging. On the one hand, the possibility of a customer-specific configuration of the products leads to high product variability and complexity in production. On the other hand, high standards of manufacturing mean that datasets are often highly skewed with very few examples of damaged parts. This makes it difficult to map all possible part variants and assembly situations in the training data of the algorithm. As modern production systems strive for zero-defect production, the appropriate composition of the data set must therefore be carefully planned and already considered in the $\mathrm{PoC}$ to estimate the robustness in series production at an early stage. ID04: "That's why the data set should be so diverse that it is precise enough for the application that you actually want to implement later, so that you can judge quite well whether it meets the requirements or accuracy that the process needs." The myriad options for vehicle customization can further slowdown the process to collect representative datasets. ID12: “We also have exotics, where parts are only installed every few thousand vehicles and if you want to store exactly such an installation condition with maybe 20 images each, then you will have a hard time getting exactly these images [...] to exaggerate, if you can get the AI algorithm to work properly with additional 10 training examples for a new part, then it is feasible. However, if you need 50.000 images for each new variant, then the question really is where to get the 50.000 images from?" To resolve this challenge, Automotive AG implemented a detailed process model to ensure that data for AI PoCs are collected in a reproducible manner. A checklist, which is used as a preliminary self-assessment tool for new PoCs estimates the 
suitability of the available data to train robust algorithms. ID06: "If a dataset is specially prepared for the $P o C$, then the reproducibility is often questionable. Therefore, we have defined a comprehensive checklist to query relevant criteria upfront." Furthermore, to handle the problem of longlasting data gathering new state-of-the-art methods to train deep neural networks get investigated. One promising method is the use of synthetic data to train algorithms. By using sophisticated digital rendering tools, training images are generated from CAD images. This decreases the need for real world image gathering. Third, we observed a data labeling challenge that is rooted in the context sensitivity of AI. As the AI output is only as good as the data the context provides, this directly links to data quality. Data labeling can often be outsourced to third-party suppliers if generic problem solving is needed, which does not require extensive domain knowledge. One prominent example is street-scene segmentation, which is needed for Autonomous Driving tasks. In the case of Automotive AG, labeling very specific manufacturing datasets requires profound knowledge and experience of the domain's context, because, e.g., material defects can be hard to spot for an untrained observer. Consequently, labeling the images by domain experts results in a higher quality of the dataset. ID04: "It was a huge effort [in our PoC] to look through all the images. I had to exactly explain to the labeler how to label the images. One could see a difference in data quality because the labelers were from our press-shop and had extensive knowledge of the process. " From a different stance, labeling images by domain experts can lead to quality issues if they must fulfill the labeling tasks beside their main duties, which are critical for keeping operational production lines running. ID04: "I once had the data labeling done by my colleagues from the technology department. The labeling was not very consistent. Some images were labeled very detailed and others very rough. Also, there were things labeled which were actually no cracks at all." To resolve this challenge, Automotive AG qualified a large team of internal factory workers as data annotators. The internal labeling team consists of domain experts with years of work experience in the manufacturing industry. This ensures that the context sensitivity aspect of AI PoCs is addressed. Data quality is further ensured by including expelled domain experts in the labeling process to perform quality checks. Before sending a labeling assignment to the internal labeling crowd a detailed labeling guide is created in an iterative manner. For that purpose, a draft labeling guide together with a data-subset - the so-called "gold standard" - gets send to multiple experts. Next, relevant metrics are calculated automatically based on the consensus of the experts. If metrics are not met yet, the labeling guide gets refined. This process runs iteratively until the required quality is achieved.

4.2.2 Process Alignment. First, we find that problemcentricity is key to motivate AI PoCs. This is rooted in the black box characteristics of AI. People tend to initiate PoCs for the sake of promoting a novel technology, which is particularly significant in view of the AI hype. ID03: "So I think you have to put the brakes on the hype a bit and simply question again whether everything really has to be solved with AI." The hype-induced bounded rationality needs to be mitigated in AI PoCs by embedding it in a clear and realistic business scenario. Our case context shows that in the field of image recognition and Computer Vision, the use of camera and sensor technology is often sufficient. Therefore, Automotive AG is challenging the necessity of AI systems in this area and, so to speak, puts AI into competition with classic approaches during a PoC. ID04: "You don't deploy AI for the sake of AI, because in the end it's just a means to reach the goal what should always be in the foreground of such a PoC-to have a goal that you want to achieve or a problem that you want to solve. And the AI is then only a means to an end." One success factor that was frequently mentioned during the case interviews is that motivation must come from a concrete problem. PoCs that focus on AI (and often prominently feature the term "AI" in their project title) are often questionable. Here, valuable problemsolution pairs need to be developed, challenged, and validated in terms of feasibility. In the manufacturing context, lean principles are therefore still essential and process orientation is the basis to define AIappropriate problems. To resolve this challenge, Automotive AG uses the early AI PoC phase to substantiate the suitability assumption for an AI technology in view of both the given context and the intended objectives. ID03: "Detecting cracks in the press shop is a very old topic. It's been around for tens of years and a wide variety of technologies has already been tested, but the technological limits to date have always been reached relatively quickly. From that stance, the idea arose that we could try out whether AI can really recognize these cracks automatically." To this end, work is carried out from the outset in heterogeneous teams comprising domain experts and AI technology experts, keeping in mind the principle that is reflected in ID10: "So I see added value for production less in the AI itself and more in the use cases that then improve production. You don't solve any problems with pure AI itself." Second, we could observe in all PoCs that we investigated at Automotive 
AG that the context sensitivity of AI makes a rigorous business process mapping inevitable. In contrast to Big Data Analytics (where the focus is on gaining knowledge from data), AI capabilities allow for automating complex tasks and processes. Therefore, it is important to first make sure that there is a formal understanding of the process itself - what inputs get passed into a process and how these get processed to form an output. In some cases, formal process models do not follow the actual practice, as manufacturing processes get optimized over time without detailed documentation. Then, it is important to properly record the process first before approaching the use of AI. ID08: "I would have looked at the entire process from front to back and then said if the process is not followed, then an AI will not solve your problem. So, you first must get the process clean. And if the claim is still that we're not getting anywhere here, then you can think about some AI or camera or something." To resolve this challenge, Automotive AG encounters the necessity to first put a particularly strong focus on processes analysis. By requiring distinct information from the outset, e.g., what are process inputs for decision making and what are the outputs, it can be ensured that the process gets formalized properly, hence the future AI assisted process can be implemented successfully. Third, we encountered the need for successful AI PoCs to be translated to a mathematical problem definition. This is rooted in the experimental character of $A I$, which requires the problem to be described in quantitative terms. This is especially tangible in the field of Computer Vision. If the task is to identify parts, then the problem can be described as a classification task. If the task is to locate paint defects on a car body, then one could map the problem as a segmentation- or localization task. ID06: "Basically, the business problem must first be translated into an analytical question, and then, of course, the question whether the available data is fundamentally suitable for the purpose and whether it is available in the required quantity and quality has to be resolved." This problem definition is crucial to various other fields as apparent in the quote above. Not only is the algorithm choice dependent on how the problem is described but also the collection and annotation must be aligned. To resolve this challenge, Automotive AG encountered the need to involve AI experts early in the stage of AI PoC definition in a consulting role. This ensures that user stories for new AI PoCs are described in a way which is comprehensible for Machine Learning Engineers. Also, problems are subdivided into discrete categories to further streamline the evaluation process.
4.2.3 Value Orientation. First, there needs to be a sound decision-making regarding the right level of use case simplification within the AI PoC. This is especially relevant as the context sensitivity of AI requires organizations to decide how to simplify complex manufacturing tasks and processes for the AI PoC to avoid the cost of a full-scale productive solution on the one hand, but on the other hand to be able to make a statement about the technical feasibility, i.e., to grasp relevant data about the task at hand and its contextual factors to account for transferability of PoC insights to a live deployment. ID01: "Of course, one cannot say ex-ante without having to spend time and effort, whether an idea really can be implemented with given requirements. So, I basically can't really get around data annotation, and I also must think about what is state of the art, and which algorithms are suitable. And that means it is important to implement the PoCs at a certain level of maturity." To resolve this challenge, Automotive AG puts great emphasis on defining the actual technicalmathematical question or hypothesis to be retrieved within the PoC. ID01: "In any case, it makes sense to really ask yourself at the beginning of the PoC what might be these critical aspects which I want to challenge or proof? Sometimes, I perceive a mindset like how to make a PoC as pleasant as possible or how to make it a success in any case? PoCs which primarily act as a marketing tool tend to focus on fancy looking UI or some sort of interactivity. This might be great for the short term, but likely not useful to solve the actual AI question." Second, the business case unpredictability is rooted in the experimental character of AI. As a result, the business case is not deterministically predictable before the PoC. Even after completion, it is usually not possible to make a conclusive statement, as the future algorithm performance can only be roughly estimated. Furthermore, the effort to rapidly integrate evolving technologies into a stability-centric IT landscape can provoke unforeseen costs. ID04: "Because although we knew that the current non-AI solution did not work well, we did not know exactly how high the costs incurred would be. So, part of the PoC was to determine this in collaboration with the Controlling department. In addition, it was important to have a certain basic understanding of AI, to estimate the effort to further tune the algorithm for a companywide scaling." To resolve this challenge, AI projects at Automotive AG are developed in an agile manner, especially in early phases. User stories in the beginning of an AI PoC typically focus on quantifying the business case. The results can then be used to derive performance requirements for an algorithm. User stories in a later stage can then customize the 
requirements for data acquisition and algorithm development. Third, the experimental character of AI requires a definition of success metrics with a strong mathematical focus. In contrast to other PoCs, success in AI projects is not immediately tangible. In many interviews, it became clear that defining the mathematical metrics and interpreting them is a challenge. To assure that the anticipated value also transfers to the real manufacturing context, the context sensitivity of AI requires the definition of a metric that is transferable to the actual problem context. This transferability challenge between measuring success in the PoC phase and later project phases is emphasized by ID01: "Evaluating AI PoCs is not trivial. The performance metrics can be difficult to understand. Especially in the field of Computer Vision you need to make sure that customers understand how exactly you evaluate whether an algorithm is working properly. This ensures that it meets their individual requirements." To resolve this challenge, Automotive AG produced a comprehensive guide for potential internal customers on how typical AI PoC metrics are calculated. This ensures that there is a common ground of understanding between AI experts and the domain experts when evaluating PoCs.

4.2.4 AI Empowerment. First, we found that due to the black box characteristics of AI, and the fuzziness of the AI term, there is a fundamental need for demystification of AI across the organization. This is also reflected in our case context, which reveals that "[t]he main problem is that most people don't know whether AI can work or not. Many also have far too high expectations. Many don't even know what it can do, and some think it can do everything and automates itself" (ID02). At Automotive AG, it is therefore important to convey a basic understanding of AI as a digital technology in a still very physical production environment. This enables employees to set proper expectations for AI. At Automotive AG, therefore, on an organizational level, different AI training courses are offered to the employees according to their level of knowledge, and use cases are presented and discussed in technology talks. During AI PoCs, technological empowerment of involved stakeholders is ensured. ID11: "In a common PoC you physically install a system to try whether something works. What makes it special for me in the presence of AI is that, if you think of image processing, you don't see the actual AI that runs behind it." To resolve this challenge on a project level, it is essential to create a common understanding of AI as early as the definition phase of the AI PoC to leave no room for speculation and to place the project on a technically sound basis. Consequently, the fuzzy term gets more tangible and trustworthy. ID04:
"Although I think it is always important to involve people, it is especially important for AI. The special thing about AI is that people understand it in very different ways, and sometimes have very wild ideas. I always came up with a few standard answers to explain to them what AI can do. In other words, to explain it in one or two sentences, always in a very basic way." Second, due to the AI learning requirements, a higher rate of customer involvement in technical tasks is required in $\mathrm{AI}$ PoCs, what relates to customer enablement. As it is the goal in an AI PoC to digitize human intelligence and collective knowledge, the department of the internal customer must take on important tasks for this. This includes, e.g., to actively participate in data annotation, and to describe the focal processes. A key success factor here is a basic understanding of AI learning requirements on the part of the context specialists so that the importance of qualitative data and how labeling influences the algorithm quality becomes comprehensible. ID05: "So to speak, the democratization, i.e., to bring that AI knowledge into the field and explain it in simple language to people who are non-specialized in AI. That's a big challenge." To resolve this challenge, Automotive AG involves subject matter experts at an early stage of an AI PoC, who stand by as advisors as the use case progresses. ID04: "So it helped a lot to have an AI expert tell me what is feasible with AI, to get an understanding of what is feasible and what is rather unrealistic. Of course, it was helpful that I was able to assess this so that I could explain it to people who are even further away from AI." Third, the black box characteristics of AI require internal capability building to be able to evaluate the AI technology potential for the corporate application context, and to assess its value in comparison to other technologies. This challenge is multiplied by both a lack of platforms and infrastructure, and a lack of internal Machine Learning skills, and difficulties of accessing talent on the market. Against the backdrop of the AI hype, it is important to be able to estimate expenses associated with $\mathrm{AI}$ and to be able to evaluate $\mathrm{AI}$ in comparison to other solutions; often even "classic" data analysis tools offer great added value so that one does not necessarily need to "shoot at a sparrow with a cannon'. This is brought to the point by ID03: "From my point of view, we simply have to gather experience now and then also be able to categorize all these requests that we are currently receiving from the manufacturing areas - whether it really makes sense to solve such a problem with AI or not, whether something is basically feasible or not." To resolve this challenge, the Automotive AG has installed innovation hubs, which explore AI among other data- 
based technologies, and explore and test the technology under near-production conditions. ID09: "By analogy with 'don't trust any statistics that you haven't falsified yourself', I would state today 'don't trust any AI PoC that you haven't set up yourself.",

\section{Recommendations for Action}

Based on the case findings, we provide four actionable recommendations for project leaders who plan to realize AI PoCs in the manufacturing context:

Prepare the manufacturing organization for data utilization at scale. Encountering data assessment challenges requires manufacturing companies to provide a connected data infrastructure and establish data governance that allows for data collectability. Moreover, qualitative training data was rated higher for project success than tuning the algorithm, as evidenced by the large portion of challenges identified in this domain.

Thus, project leaders should induce the necessary changes into organizational structures and IT landscapes through top management buy-in. Two areas in particular stand out here. First, the deployment of a manufacturing data platform for collecting data from different production plants in a harmonized and consistent manner are advisable to facilitate efficient and effective AI PoCs. Second, decision makers should establish a domain-focused labeling team preferably from internal sources - to ensure a high standard of data quality.

Make complex manufacturing processes transparent and upskill yourself. Encountering process alignment challenges requires manufacturing companies to take a process-oriented approach to problems, which, after process recording, ultimately enables a mathematical problem definition.

Thus, project leaders should question the contextual problem with the greatest care in the early AI PoC phase and incorporate AI experts early to ensure the problem transfer into an AI-appropriate structure. Therefore, the project manager should have contextual understanding of manufacturing and acquire basic knowledge of state-of-the-art AI technologies to moderate between the parties in crossfunctional teams.

Ensure value orientation by establishing a PoC Takeoff and Landing checklist. Encountering value orientation challenges requires manufacturing companies to manage an AI portfolio, which covers scalable AI solutions and strategically relevant manufacturing aspects, based on vague assumptions that stem from AI PoCs.

Thus, project leaders should promote the agile project procedure in AI PoCs and define start and stop criteria that are regularly adjusted to the AI portfolio strategy. The three focal areas of this list should be (1) the presence and formulation of quantitative success metrics (2) termination criteria and (3) the critical aspects which the AI PoC wants to prove. Project leaders should draw particular attention to the third aspect and assess which individual characteristics of their company context could hinder the success of the AI idea.

Use AI PoCs as a communication instrument to educate and empower the manufacturing organization. Encountering AI empowerment challenges requires manufacturing companies to send a clear signal to its employees that they want to make $\mathrm{AI}$ accessible to them and that everyone can make an important contribution to its use.

Thus, project leaders should ensure that their AI prototypes get communicated so that the AI phenomenon becomes tangible across the organization - following the principle "show don't tell". Especially in the physical manufacturing context, it is valuable to promote AI "in action" to explore new application ideas in discussion with domain experts or even to be able to scale one's own project.

\section{Concluding Remarks}

In this study, we empirically identified challenges in AI POCs in the context of a manufacturing company. Our results illustrate how the specific characteristics of AI give rise to these challenges. Based on that we provide four recommendations for building PoCs successfully in manufacturing contexts: (1) prepare the manufacturing organization for data utilization at scale, (2) make complex manufacturing processes transparent and upskill yourself, (3) ensure value orientation by establishing a PoC Takeoff and Landing checklist, and (4) use AI PoCs as a communication instrument to educate and empower the manufacturing organization. Our study has highlighted opportunities of AI PoCs in the manufacturing context. Even within technology-savvy organizations there is still a bridge to gap when implementing and scaling AI solutions, which should encourage further research to contribute to this emerging and increasingly relevant stream of research.

\section{References}

[1] Dwivedi, Y. K., Hughes, L., Ismagilova, E., Aarts, G., Coombs, C., Crick, T., Duan, Y., et al. 2021. Artificial Intelligence (AI): Multidisciplinary Perspectives on Emerging Challenges, Opportunities, and Agenda for Research, Practice and Policy. International Journal of Information Management 57 (April): 101994. 
[2] Cram, W. A., and Wiener, M. 2020. Technologymediated Control: Case Examples and Research Directions for the Future of Organizational Control. Communications of the Association for Information Systems, 46(4), pp. 70-91.

[3] Davenport, T. H. 2018. From Analytics to Artificial Intelligence. Journal of Business Analytics, (1:2), Taylor \& Francis, pp. 73-80.

[4] von Krogh, G. (2018). Artificial Intelligence in Organizations: New Opportunities for PhenomenonBased Theorizing. Academy of Management Discoveries, 4(4), pp. 404-409.

[5] Benbya, H., Pachidi, S., and Jarvenpaa, S. L. 2021. Special Issue Editorial: Artificial Intelligence in Organizations: Implications for Information Systems Research. Journal of the Association for Information Systems, 22(2), pp. 281-303.

[6] Rai, A., Constantinides, P., and Sarker, S. 2019. Editor'S comments: next-generation digital platforms: toward human-AI hybrids. MIS Quarterly, 43(1), pp. iii-x.

[7] Lacity, M., \& Willcocks, L. 2021. Becoming Strategic with Intelligent Automation. MIS Quarterly Executive, 20(2), pp. 1-14.

[8] Brynjolfsson, E., \& Mitchell, T. 2017. What can machine learning do? Workforce implications. Science, 358(6370), pp. 1530-1534.

[9] Goasduff, L. 2019. Top Trends on the Gartner Hype Cycle for Artificial Intelligence. Gartner. https://www.gartner.com/smarterwithgartner/toptrends-on-the-gartner-hype-cycle-

[10] Kaplan, A., and Haenlein, M. 2019. Siri, Siri, in my hand: Who's the fairest in the land? On the interpretations, illustrations, and implications of artificial intelligence. Bus. Horizon., 62(1), pp. 15-25.

[11] Duan, Y., Edwards, J. S., and Dwivedi, Y. K. 2019. Artificial intelligence for decision making in the era of Big Data-evolution, challenges and research agenda. Int. Journal of Information Management, 48, pp. 63-71.

[12] Ransbotham, S., Khodabandeh, S., Fehling, R., La Fountain, B., and Kiron, D. 2019. Winning With AI. https://image-src.bcg.com/Images/Final-Final-ReportWinning-With-AI-R_tcm9-231660.pdf

[13] Winkler, M., Tolido, R., Thieullent, A. L., Finck, I., Buvat, J., Khadikar, A., and Hiral, S. 2019. Accelerating Automotive's AI Transformation: How Driving AI Enterprise-Wide Can Turbo-Charge Organizational Value. Capgemini Research Institute. https://www.capgemini.com/wp-content/uploads/2019 /03/30-min-\%E2\%80\%93-Report-3.pdf

[14] Canhoto, A. I., and Clear, F. 2020. Artificial intelligence and machine learning as business tools: A framework for diagnosing value destruction potential. Business Horizons, 63(2), pp. 183-193.

[15] Hofmann, P., Jöhnk, J., Protschky, D., and Urbach, N. 2020. Developing Purposeful AI Use Cases - A Structured Method and its Application in Project Management. $15^{\text {th }}$ International Conference on Wirtschaftsinformatik, pp. 33-49.

[16] Engel, C., van Giffen, B., and Ebel, P. 2021. Empirically Exploring the Cause-Effect Relationships of AI Characteristics, Project Management Challenges, and Organizational Change. Wirtschaftsinformatik 2021 Proceedings. 3.

[17] Amigoni, F., and Schiaffonati, V. 2018. Ethics for robots as experimental technologies: Pairing anticipation with exploration to evaluate the social impact of robotics. IEEE Robot. Autom. Mag. 25, pp. 30-36.

[18] Lieberman, H., and Selker, T. 2000. Out of context: Computer systems that adapt to, and learn from, context. IBM Syst. J. 39, pp. 617-632.

[19] Castelvecchi, D. 2016. Can we open the black box of AI? Nature. 538, pp. 20-23.

[20] Jordan, M.I., and Mitchell, T.M. 2015. Machine learning: Trends, perspectives, and prospects. Science. 349, pp. 255-260.

[21] George, G., Haas, M. R., and Pentland, A. 2014. Big Data and Management. Academy of Management Journal, 57(2), pp. 321-326.

[22] Bednar, P. M., and Welch, C. 2020. Socio-Technical Perspectives on Smart Working: Creating Meaningful and Sustainable Systems. Information Systems Frontiers, 22(2), pp. 281-298.

[23] Loureiro, S. M. C., Guerreiro, J. and Tussyadiah, I. 2021. Artificial Intelligence in Business: State of the Art and Future Research Agenda. Journal of Business Research. 129, pp. 911-26.

[24] Neto, A. J. R., Borges, M. M., and Roque, L. 2018. A Preliminary Study of Proof of Concept Practices and their Connection with Information Systems and Information Science. Proceedings of the Sixth International Conference on Technological Ecosystems for Enhancing Multiculturality, pp. 1-6.

[25] Nunamaker, J. F., Briggs, R. O., Derrick, D. C., and Schwabe, G. (2015). The Last Research Mile: Achieving Both Rigor and Relevance in Information Systems Research. Journal of Management Information Systems, 32(3), pp. 10-47.

[26] Oxford English Dictionary. 2014. Oxford Univ. Press.

[27] Kendig, C. E. 2016. What is Proof of Concept Research and how does it Generate Epistemic and Ethical Categories for Future Scientific Practice? Science and Engineering Ethics, 22(3), pp. 735-753.

[28] Bennett, N., and Lemoine, J. 2014. What VUCA really means for you. Harvard Business Review, 92(1/2).

[29] Recker, J. 2012. Scientific Research in Information Systems: A Beginner's Guide. Springer Science \& Business Media.

[30] Yin, R. K. 2003. Case Study Research Design and Methods. Applied Social Research Methods Series.

[31] Lasi, H., Fettke, P., Kemper, H.-G., Feld, T., and Hoffmann, M. 2014. Industry 4.0. Business \& Information Systems Engineering, pp. 239-242.

[32] Demlehner, Q., Schoemer, D., and Laumer, S. 2021. How can artificial intelligence enhance car manufacturing? A Delphi study-based identification and assessment of general use cases. International Journal of Information Management, 58, pp. 1-14.

[33] Corbin, J. M., and Strauss, A. 1990. Grounded theory research: Procedures, canons, and evaluative criteria. Qualitative Sociology, 13(1), 3-21. 